\title{
Téoros
}

Revue de recherche en tourisme

\section{Tourisme et infections transmises sexuellement}

\section{Dominique Tessier}

Volume 24, numéro 3, automne 2005

Tourisme et santé, quelques perspectives

URI : https://id.erudit.org/iderudit/1071076ar

DOI : https://doi.org/10.7202/1071076ar

Aller au sommaire du numéro

Éditeur(s)

Université du Québec à Montréal

ISSN

0712-8657 (imprimé)

1923-2705 (numérique)

Découvrir la revue

Citer cet article

Tessier, D. (2005). Tourisme et infections transmises sexuellement. Téoros, 24(3), 19-24. https://doi.org/10.7202/1071076ar d'utilisation que vous pouvez consulter en ligne.

https://apropos.erudit.org/fr/usagers/politique-dutilisation/ 


\section{Tourisme et infections transmises sexuellement}

\section{Dominique Tessier}

Partout dans le monde, à chaque année, dans les pays en développement et dans les pays industrialisés, un nombre croissant d'enfants sont vendus ou embarqués dans un réseau de prostitution pour une clientèle, elle aussi en croissance, à la recherche de plaisirs sexuels maintenant reconnus comme des crimes ${ }^{1}$. Le Fonds des Nations unies pour l'enfance (Unicef) estime qu'un million d'enfants, dont une grande majorité de filles, sont embarqués de force chaque année dans le commerce du sexe. Selon I'ONUSIDA, il semble que les filles soient recrutées de plus en plus jeunes pour l'industrie du sexe, en partie parce que l'on croit, à tort, que les toutes jeunes filles ont peu de chances d'être contaminées par le virus du $\mathrm{VIH} / \mathrm{SIDA}$. Dans certains pays d'Afrique, la rumeur locale prétend qu'un homme infecté qui a des relations sexuelles avec une jeune fille vierge se « décontamine ${ }^{2}$.

Cette forme de tourisme sexuel est si répandue et accessible qu'elle constitue une grande part du tourisme qui fréquente plusieurs pays, dont Madagascar, où l'on vient pour le pratiquer sans aucune répression par les autorités. Ce tourisme sexuel se développe rapidement et, pour répondre aux demandes des clients, peut revêtir plusieurs formes, dont certaines particulièrement sordides s'en prennent à des enfants de plus en plus jeunes ${ }^{3}$.

Albert est un touriste de 57 ans qui prépare un voyage de quatre à huit semaines en Thailande. Il part seul et compte habiter exclusivement dans des régions touristiques, peut-être même seulement Bangkok s'il trouve rapidement à s'y établir confortablement. En consultation à la clinique santé-voyage, il refuse toute

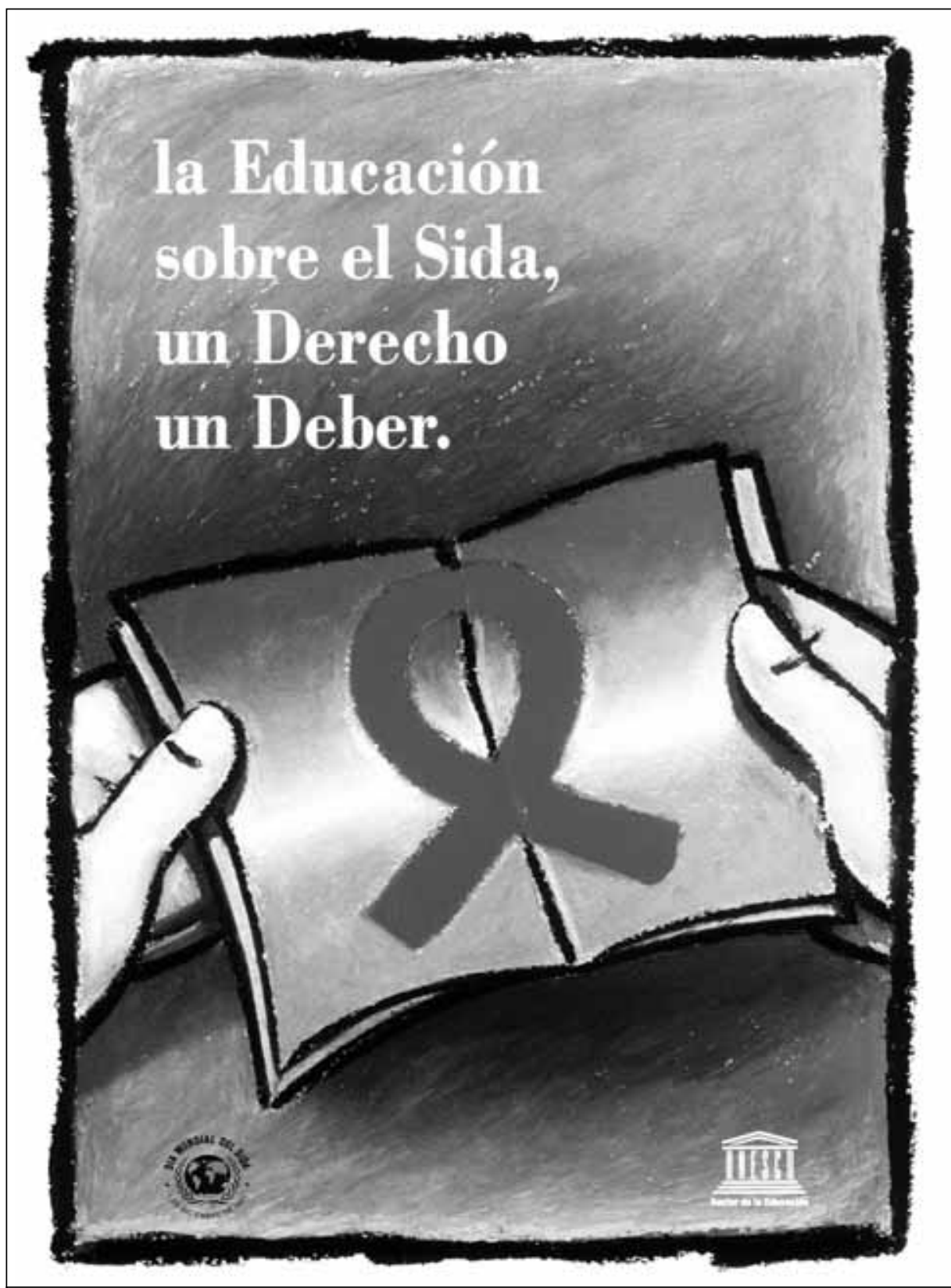

Affiche - Éducation, prévention contre le SIDA. Photo : @ UNESCO 
médication contre le paludisme, mais désire une grande quantité de ciprofloxacine, un antibiotique. Vous lui parlez de résistance possible et il est tout heureux que vous lui suggériez aussi du zythromax en deuxième ligne. Puis, en hésitant un peu, il vous demande si ces deux médications seront vraiment suffisantes et efficaces contre les infections transmises sexuellement (ITS) en Thaillande. Vous le questionnez un peu plus... Comme vous n'avez pas sauté au plafond, il se confie : il souhaite faire des bonnes œuvres en Thaillande. II achètera une très jeune fille (pas de risque de sida) dans un village qu'il paiera avec assez d'argent pour faire vivre sa famille pour au moins un an (soit de 2 à $5 \$ C A D$ par jour). À la fin du voyage, responsable, il la ramènera à ses parents et ils vivront heureux... jusqu'à l'année prochaine. Il demandera à la jeune fille de faire la cuisine, les courses et le ménage et, bien sûr, de réchauffer son lit. II est vieux, mais pas fini.

\section{Mises en situation}

Dans l'esprit de bien des gens, le tourisme sexuel fait référence à des hommes, surtout vieux mais de plus en plus jeunes, pour qui le but ultime de tout voyage est d'avoir des relations sexuelles avec de jeunes partenaires, garçons et filles. Ceux qui s'y adonnent et leurs victimes se rencontrent dans quelques destinations soleil bien connues et réputées pour ce genre de services. Et même si personne ne souhaite un tel travail pour ses propres enfants, plusieurs acceptent facilement la fable voulant que les revenus obtenus par ces enfants (ou de leur achat par des entremetteurs) permettent à de nombreuses familles villageoises d'éviter la misère. Si ces enfants sont effectivement parfois recrutés avec promesse d'éducation ou de "salaire " imposant, très peu leur est versé des milliards de dollars brassés par cette industrie.

Mais les lois internationales changent. Le 9 octobre 2000, en France s'est ouvert le procès d'un français de 47 ans accusé d'agression sexuelle sur une fillette de 11 ans en Thaillande lors de "vacances sexuelles ». II s'agit de la première personne à être amenée à procès en France depuis la Loi sur les crimes reliés au tourisme sexuel en $1994^{4}$.
Si cette réalité existe et doit être identifiée, nommée, décriée et criminalisée, elle ne doit pas occulter une autre réalité liée à la sexualité lors d'activités touristiques ou de voyages.

Serge prépare un voyage de trois mois en République démocratique du Congo. /l y a travaillé pendant plusieurs semaines par année depuis trois ans à la mise sur pied d'un système national de communications avant de prendre sa retraite. Sa femme devant encore travailler deux ans avant sa propre retraite, il a décidé de passer le temps en offrant ses services à un organisme non gouvernemental et de retourner dans ce pays qu'il connait bien et apprécie. Ce que sa femme ignore, c'est qu'il y retrouvera son autre "femme ", une jeune congolaise qui l'avait suivi un soir après l'avoir servi au restaurant. Elle avait frappé à sa porte "C'est l'amour, monsieur» et il avait ouvert. Curiosité et ébriété mêlées. II ne s'était pas protégé, mais elle avait juré être toujours fidèle. II s'est quand même un peu inquiété lorsqu'elle a admis que son mari, lui, ne l'était pas.

Irma, revient tout juste de Sainte-Lucie. Elle débarque sans rendez-vous à la clinique des voyageurs, aussitôt arrivée, et insiste pour voir un médecin. Devant son air paniqué, la secrétaire accepte de vérifier si un médecin peut la recevoir en surplus ce matin-là. Elle entre dans le bureau de consultation, valise à la main, très bronzée, mais visiblement au bord des larmes. La femme de chambre, visiblement émue par le généreux pourboire qu'elle lui laissait, lui a dit que l'instructeur de plongée avec qui elle avait passé plusieurs nuits avait le sida. Irma ne sait plus si elle s'est protégée lors de toutes ses relations. Il y en a tant eu en trois jours. Irma n'avait pas eu de partenaires sexuels depuis sept ans. Les hommes québécois ne semblent pas attirés par ses rondeurs.

Denis est un homme d'affaires. Cadre dans une grande multinationale, il voyage régulièrement en Asie et en Amérique du Sud. Jeune et en bonne santé, séjournant dans les meilleurs hôtels et se déplaçant toujours en classe affaires, il n'a jamais vraiment été convaincu de la nécessité de consulter une clinique santévoyage. Sa mère lui avait tenu la main lorsqu'il avait reçu son dernier vaccin, vers sept ans. Aucun autre depuis. II a ré- cemment réussi "un coup en or» en Thaillande et en a profité pour prendre quelques jours de congé sur place. Seul. Les masseuses étaient à la hauteur de ses fantasmes. Mais le réveil a été douloureux quand il a ressenti des brûlures mictionnelles et constaté un écoulement urétral le lendemain de son retour. II a eu des relations, non protégées, avec sa fiancée. Maintenant, il n'en dort plus. Il a peur non seulement d'avoir été infecté, mais, en plus, d'avoir transmis cette infection.

André aussi est un homme d'affaires, mais pour une toute petite compagnie qui grandit rapidement. Il a dû se rendre au Brésil en catastrophe pour conclure les démarches amorcées par un collègue rapatrié à la suite d'un infarctus. Mais il a gagné le pari et signé le gros contrat avec le groupe local, malgré son peu de connaissances des habitudes et des rituels. II a suivi ses hôtes pour aller fêter dignement cette signature. Aux frais $d u$ client. Bar, alcool coulant à flot et jolies femmes sur ses genoux, il se souvient vaguement avoir eu peur que ça aille trop loin. Le lendemain, au réveil, une jeune femme était toujours au lit, avec lui, mais il n'arrivait pas à se souvenir ce qui s'était passé. Trop mal à l'aise pour chercher les mots en portugais, il est reparti pour Montréal et a tout dit à sa femme qui l'attendait à l'aéroport. Les voici tous les deux qui consultent à la clinique post-exposition pour des conseils et un traitement préventif.

Ces personnes ne représentent que quatre maillons dans la longue chaîne de transmission du sida et des infections transmises sexuellement associées aux voyages et au tourisme sexuel. Toutes ont eu des relations sexuelles avec de nouveaux partenaires pendant leur voyage. Toutes n'ont utilisé aucune protection. Toutes avaient reçu des conseils concernant les risques liés aux relations sexuelles, à la prévalence du virus de l'immunodéficience humaine, de l'hépatite $B$ et d'autres infections transmissibles sexuellement dans les pays où ils se rendaient. Toutes ont distraitement écouté ces conseils comme s'ils s'adressaient à d'autres.

André ne s'est pas présenté dans une clinique pour les voyageurs et n'a pas consulté son médecin avant tous ses voyages. Sa secrétaire avait toutefois pris la précaution d'appeler dans une clinique des voyageurs. On lui avait fait parvenir de la documentation 
sur les différents risques et, un peu à la blague, sa secrétaire avait surligné les passages sur les risques de contacts sexuels. On lui avait fortement recommandé la vaccination contre les hépatites $\mathrm{A}$ et $\mathrm{B}$ et une mise à jour de ses vaccins de base, mais il n'avait pas trouvé le temps de se rendre dans une clinique. Par contre, faut-il souligner que même les voyageurs qui le font ne se font pas offrir systématiquement la vaccination contre I'hépatite $B$, même pour des séjours prolongés en pays hyper-endémique (Poland et al., 1996). Cette vaccination est pourtant recommandée pour la plupart des Canadiens, indépendamment du contexte de voyage. Même si les consultations dans des cliniques santé-voyage sont en augmentation au Canada (MacDougall et al., 2001) la plupart des voyageurs reçoivent peu ou pas de conseils concernant les risques d'exposition au sang ou aux liquides biologiques et, notamment, aux relations sexuelles sans protection avec de nouveaux partenaires.

Berthe-Louise, 47 ans, part la semaine prochaine rejoindre son mari à Prague. Celui-ci est en mission en Bosnie depuis six mois. Âgé de 53 ans, il est en très bonne santé et ne présente, selon elle, aucune allergie ou problème de santé. Elle insiste bien avant de vous demander une prescription de Viagra pour lui : dans ses derniers courriels, il mentionnait qu'il avait l'impression de ne plus avoir de libido depuis son arrivée dans ces régions ravagées. Comme elle n'est pas encore ménopausée et pas faite en bois, elle pense que vous pourriez l'aider.

Ce sont habituellement les hommes qui demandent des stimulants de la fonction érectile avant un voyage. Cette demande est un peu inhabituelle et peu sembler anodine, mais requiert la plus grande prudence. Un médecin ne doit pas compléter une ordonnance pour une personne qui n'est pas son patient, qu'il n'a jamais rencontrée, quelle que soit la médication. Le mari de Berthe-Louise a aussi fait des rencontres pendant son séjour en mission. Mission beaucoup plus éprouvante qu'il n'avait prévu ou qu'il ne peut raconter. Alors, pour s'évader... Sachant que sa femme venait bientôt, il a laissé planer quelques allusions sur ses difficultés sexuelles, croyant ainsi ne pas avoir à utiliser un préservatif avec sa conjointe de 19 ans. Mais un médecin trop accommodant risquerait de compromettre son plan.
Nous avons mené (Correia et al., 2001), au Canada en 1996, une étude qui visait à évaluer de tels risques. De juin à octobre 1996, 900 questionnaires post-voyage ont été postés à des individus de 16 ans et plus qui avaient consulté dans une clinique des voyageurs avant leur départ. Ces voyageurs, qui prévoyaient un séjour de moins de deux mois en juillet 1996 et en mars 1997, avaient consulté soit le Centre de médecine de voyage du Québec de l'hôpital Saint-Luc à Montréal, soit la Travel and Inoculation Clinic de I'hôpital général de Toronto ou la Travel Medicine Clinic de I'hôpital St. Joseph de Hamilton.

\section{Méthodologie}

Le questionnaire a été pré-testé sur un échantillon de voyageurs avant sa finalisation et son administration. Les voyageurs n'ont pas été avisés avant leur voyage qu'ils seraient sollicités à leur retour. Les questionnaires ont été complétés anonymement. Le questionnaire comportait 26 questions couvrant les données démographiques générales, les renseignements sur le sexe et l'âge, l'occupation, le niveau d'éducation, les détails du voyage tels que destination(s), but(s) du voyage, description de(s) l'hébergement(s) et les risques associés à des relations sexuelles occasionnelles, ou encore à des expositions potentielles à du sang ou à des liquides biologiques.

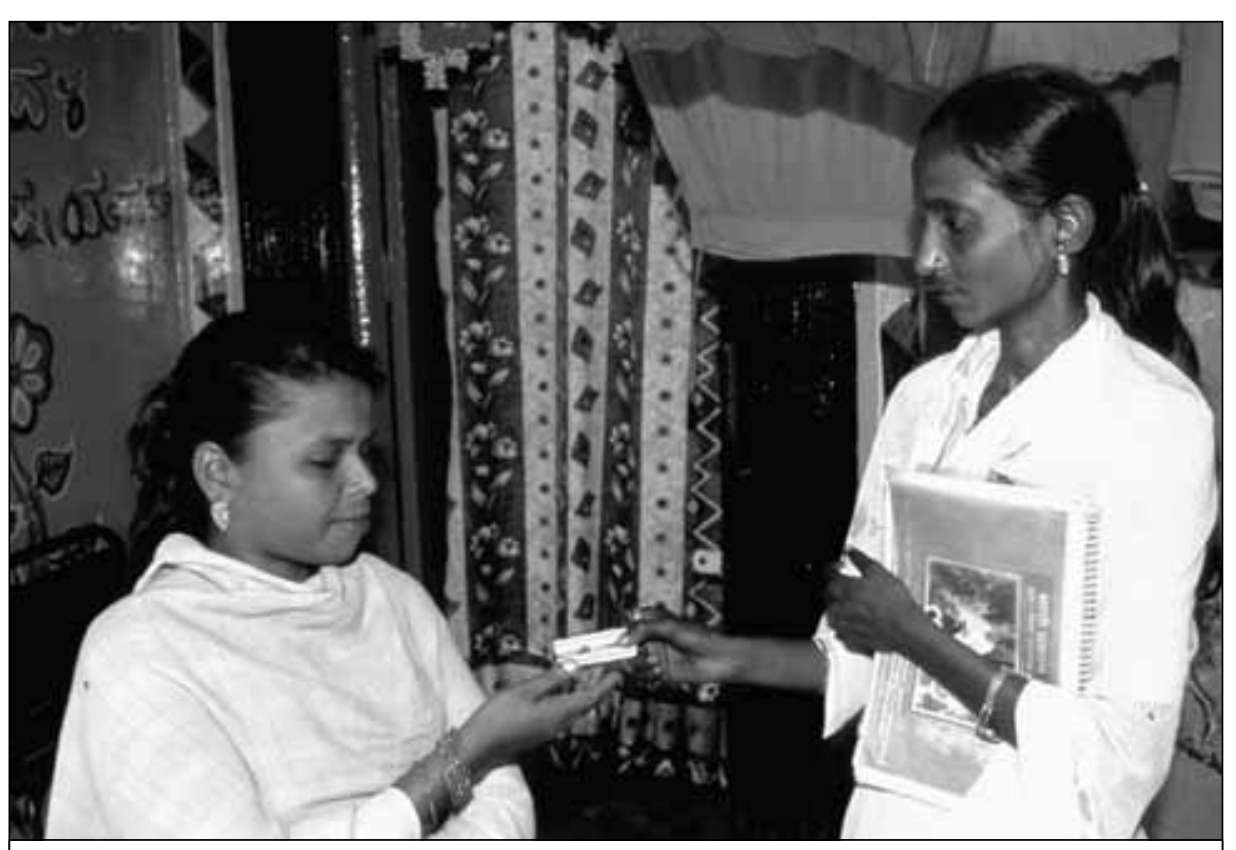

Programme de prévention (Sathi Project) destiné aux travailleuses du sexe à Bombay, Inde.

Photo: Organisation mondiale de la santé/P.Virot
L'analyse statistique a été réalisée à l'aide de Statistix 3.1 (logiciel analytique de $\mathrm{NH}$ ). Les données qualitatives ont été analysées en utilisant le test de chi carré avec la correction de Yates pour des variables discrètes et les données quantitatives en utilisant le t-test de Student normalisé ou l'essai de MannWhitney (Mann-Whitney rank sum test [two tailed] for continuous variables).

\section{Résultats}

Un total de 410 personnes (45,6\%) ont retourné le questionnaire. Parmi celles-ci, 10 personnes $(2,4 \%)$ ont retourné un questionnaire incomplet qui n'a pu être inclus dans l'analyse. Des 400 questionnaires retenus, 200 avaient été complétés par des résidants du Québec qui avaient consulté au Centre de médecine de voyage du Québec de l'hôpital Saint-Luc à Montréal. Les autres questionnaires provenaient de résidants de l'Ontario qui avaient consulté la Travel and Inoculation Clinic de l'hôpital général de Toronto ou la Travel Medicine Clinic de I'hôpital St. Joseph de Hamilton.

L'échantillon total se composait de 192 mâles et de 208 femelles. L'âge moyen de ces voyageurs était de 41,0 ans $(95 \%, \mathrm{Cl}$ 40-43). Tous les répondants étaient des résidants canadiens; 76 (19,0\%) n'étaient pas nés au Canada. Bien que 24 voyageurs $(5,9 \%)$ étaient des travailleurs dans un 
secteur médical (médecin, infirmière, chiropraticien ou technologue de laboratoire), le but du voyage dans ce groupe se limitait au tourisme. Par ailleurs 321 voyageurs (80,2 \%) avaient une éducation de niveau universitaire (bachelier et/ou diplôme professionnel), 36 $(9,0 \%)$ possédaient un certificat en commerce, 38 (9,5\%) poursuivaient actuellement des études à l'école secondaire ou avaient obtenu un diplôme d'études secondaires et 5 (1,3\%) n'avaient pas d'éducation formelle. La durée moyenne de voyage était de 5,7 semaines (médiane 3,0, écart $95 \%$, Cl 4.9-6.6). D'une manière prédominante ces voyageurs étaient partis pour le tourisme $(62,7 \%)$ et $78,0 \%$ étaient accompagnés d'au moins un compagnon de voyage (ami, membre de la famille, associé et/ou partenaire d'affaires). Les pays d'Asie étaient les destinations les plus fréquentées ; 120 voyageurs $(30,0 \%)$ avaient visité un pays ou plus dans ce continent. La majorité de voyageurs (83,0 \%) ont indiqué qu'ils sont restés dans un secteur urbain pour tout ou une grande proportion de leur séjour.

Trente-six (36) voyageurs $(9,0 \%)$ ont eu des relations sexuelles avec une personne autre que leur compagnon de voyage ; 14 (38,9\%) avaient projeté avant leur départ avoir des relations sexuelles avec de nouveaux partenaires pendant qu'ils voyageaient à l'étranger. Parmi ceux qui ont été sexuellement actifs (17 hommes et 19 femmes), 14 (38,9\%) l'ont été avec un voyageur à l'extérieur de leur groupe, 20 (55,6\%) avec des habitants locaux et $2(5,5 \%)$ avec des travailleurs du sexe commerciaux. Le nombre médian de partenaires était de 1,0 (moyenne 1,8, écart 1-20). L'âge moyen de ceux qui étaient actifs sexuellement était de 35,5 ans (95\%, Cl 30-40), comparé à un âge moyen de 41,6 ans pour ceux qui n'étaient pas sexuellement actifs $(p<0,001)$. Vingt-cing (25) voyageurs $(69,4 \%)$ sexuellement actifs ont indiqué qu'ils avaient utilisé un préservatif pour toutes leurs relations sexuelles. Les voyageurs qui avaient eu des relations sexuelles avec des partenaires occasionnels durant leur voyage avaient en moyenne une durée de séjour plus longue que ceux qui n'en avaient pas eu. Aussi, ceux qui avaient le "sexe désinvolte " avaient des voyages plus longs, avec une moyenne de 5,8 semaines, comparativement à 4,7 semaines pour ceux qui n'avaient pas eu de nouveaux partenaires; cependant, cette différence n'a pas atteint la signification statistique.

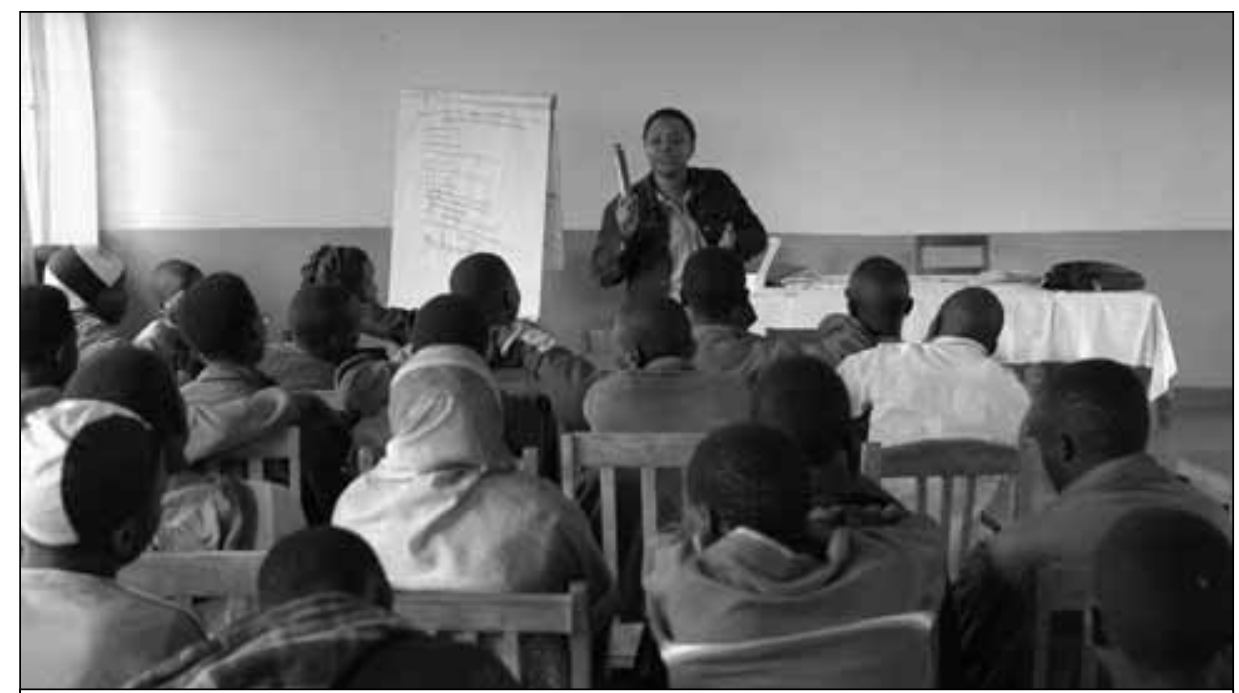

Programme de l'UNESCO pour former à la prévention contre le SIDA des enfants de la rue et des personnes en danger.

Photo: Michel Ravassard/ C UNESCO

Au total, il y a eu 77 incidents d'exposition potentielle au sang ou aux liquides biologiques, chez 60 voyageurs différents (15,0\%) (tableau 1). L'âge moyen des voyageurs de l'Ontario était significativement plus élevé que celui des voyageurs du Québec, 45 contre 37 ans ( $p=0,0000)$. II n'y avait pas de différence significative pour ce qui est de la distribution entre les deux sexes, ni de la durée moyenne de leurs voyages. Comparé aux voyageurs de l'Ontario, les voyageurs de Québec avaient une probabilité significativement plus importante de visiter l'Amérique centrale et l'Amérique du Sud (34,5\% vs 17,0\%, $p=0,0001)$. Les voyageurs de l'Ontario avaient aussi une probabilité significativement plus importante de visiter plusieurs continents (32,0\% vs 7,5\%, $p=0,0000)$. L'injection parentérale pour des raisons médicales n'était pas significativement différente entre les deux groupes. La blessure parentérale pour des traitements non traditionnels était plus commune chez les habitants du Québec $(p=0,07)$. Les voyageurs du Québec avaient une probabilité significativement plus importante de s'engager dans une activité sexuelle occasionnelle pendant leur voyage que les voyageurs de l'Ontario $(p<0,001)$. Cependant, le nombre moyen de partenaires sexuels était plus élevé parmi les voyageurs de l'Ontario (3,75 partenaires différents, comparé à 1,27 pour les voyageurs du Québec) ; cette différence n'était pas statistiquement significative. Les 8 voyageurs de l'Ontario qui se sont engagés dans une activité sexuelle occasionnelle ont affirmé avoir utilisé un préservatif lors de chaque rencontre, comparé à $61 \%$ des 28 voyageurs du Québec sexuellement actifs. Le nombre total de voyageurs du Québec qui ont été potentiellement exposés au sang ou aux liquides biologiques était significativement plus élevé que chez les voyageurs de l'Ontario (43 vs 17, $p=0,0000$ ).

Un échantillon aléatoire de $10 \%$ des non-répondants de l'hôpital de Toronto a été analysé pour déterminer s'ils étaient comparables aux répondants. Les non-répondants étaient significativement différents des répondants: ils étaient plus jeunes (l'âge moyen était de 33 ans vs 41 ans, $p=0,0000$ ) et avaient voyagé pour une période plus longue $(9,1$ semaines vs 5,7 semaines).

\section{Discussion}

L'activité sexuelle avec un nouveau partenaire était la forme la plus fréquente de comportement à risque parmi les voyageurs faisant partie de l'étude. Ainsi, $9 \%$ de nos répondants ont été actifs sexuellement avec une personne autre que leur compagnon de voyage et seulement $69 \%$ ont indiqué avoir utilisé des préservatifs. Les études du UK9 and Switzerland (Steffen et al., 1987) ont rapporté que 5 et $6,4 \%$, respectivement, des personnes revenant de voyage avaient eu des relations sexuelles avec de nouveaux partenaires pendant leur séjour à l'étranger. Bien que dans notre étude l'âge moyen des voyageurs actifs sexuellement était significativement plus bas, le sexe et la durée moyenne du voyage n'étaient pas significa- 
tivement différents de ceux des personnes qui n'ont pas eu de relations sexuelles. Soixante-dix huit pour-cent (78\%) des voyageurs (28 des 36) qui avaient eu des relations sexuelles pendant leur séjour à l'étranger étaient des habitants du Québec. Cette différence pourrait être attribuable, partiellement, à l'âge moyen plus bas des répondants de cette province.

Bien que la majorité $(55,6 \%)$ des contacts sexuels soient survenus avec des habitants locaux, 2 voyageurs (5,5\%) ont eu des relations avec un ou des travailleurs du sexe commerciaux. Un voyageur a reconnu avoir eu 20 partenaires sexuels différents, tous des prostitués. Des 36 voyageurs sexuellement actifs dans cette étude, 30 (83,3\%) ont déclaré que le but de leur voyage était le tourisme, 3 (8,3\%) étaient en voyage d'affaires et $3(8,3 \%)$ étaient en voyage pour faire du bénévolat, de la coopération ou du missionnariat. II est important de noter que presque $40 \%$ de ceux qui étaient actifs sexuellement avaient planifié, avant le départ, avoir des relations sexuelles occasionnelles pendant leur voyage. Cela confirme les résultats d'une étude récente faite en Australie qui a démontré que $68 \%$ de voyageurs souhaitaient qu'une partie de leur voyage soit consacrée à la recherche de nouveaux partenaires sexuels (Mulhall et al., 1993). Ces ré- sultats font donc ressortir l'importance que devraient accorder les cliniques santé-voyage aux conseils concernant les risques et les mesures préventives face aux infections transmises sexuellement.

En outre, les résultats exposés dans cette étude représentent vraisemblablement une sous-estimation de la réalité en ce qui a trait aux comportements à risque des voyageurs canadiens et ce, pour plusieurs raisons. Premièrement, les personnes qui ont participé à l'étude avaient toutes visité une clinique santé-voyage avant leur départ et peuvent donc représenter une population de voyageurs plus prudents. Deuxièmement, parmi les voyageurs de Toronto, les répondants étaient, en moyenne, plus vieux que les non-répondants; les voyageurs plus âgés représentent un groupe plus conservateur tant dans leurs comportements que face aux risques (Samuels et al., 1991 ; Elliott et al., 1993). De plus, les comportements à risque déclarés dans une étude rétrospective telle que la nôtre peuvent être sous-rapportés puisque les voyageurs doivent se souvenir des événements. Les résultats de notre étude suggèrent que les voyageurs à l'étranger, même pour de courtes périodes, courent un risque considérable d'exposition aux agents infectieux transmis par un contact avec le sang et les liquides biologiques. II faut

\section{Tableau 1}

\begin{tabular}{|c|c|c|c|c|c|c|}
\hline \multicolumn{7}{|c|}{ Facteurs de risque associés à une exposition à du sang ou à des liquides biologiques } \\
\hline \multirow[b]{3}{*}{ Facteur de risque } & \multicolumn{6}{|c|}{ Nombre de voyageurs } \\
\hline & \multicolumn{2}{|c|}{$\begin{array}{l}\text { Ontario } \\
(N=200)\end{array}$} & \multicolumn{2}{|c|}{$\begin{array}{l}\text { Québec } \\
(N=200)\end{array}$} & \multicolumn{2}{|c|}{$\begin{array}{l}\text { Total } \\
(N=400)\end{array}$} \\
\hline & n & $\%$ & n & $\%$ & n & $\%$ \\
\hline $\begin{array}{l}\text { Injection parentérale pour des } \\
\text { raisons médicales (ex. : médication) }\end{array}$ & 7 & 3.5 & 6 & 3.0 & 13 & 3.2 \\
\hline $\begin{array}{l}\text { Blessure parentérale pour des traitements } \\
\text { non traditionnels (ex.: acupuncture) }\end{array}$ & 0 & 0.0 & 4 & 2.0 & 4 & 1.0 \\
\hline Récréationnel (ex. : tatouage, piercing) & 0 & 0.0 & 2 & 1.0 & 2 & 0.5 \\
\hline Blessure avec abrasion & 0 & 0.0 & 2 & 1.0 & 2 & 0.5 \\
\hline $\begin{array}{l}\text { Partage d'instruments d'hygiène personnelle } \\
\text { (ex. : rasoir, brosse à dents) }\end{array}$ & 3 & 1.5 & 17 & 8.5 & 20 & 5.0 \\
\hline Activités sexuelles** & 8 & 4.0 & 28 & 14.0 & 36 & 9.0 \\
\hline Usage de drogues IV & 0 & 0.0 & 0 & 0.0 & 0 & 0.0 \\
\hline $\begin{array}{l}\text { Total des voyageurs qui ont été exposés } \\
\text { à au moins un facteur de risque }\end{array}$ & 18 & 9.0 & 42 & 21.0 & 60 & 15.0 \\
\hline \multicolumn{7}{|l|}{$\begin{array}{l}* p=0,07 \\
* * \quad p<0,001\end{array}$} \\
\hline Source : Données recueill & & & & & & \\
\hline
\end{tabular}

conséquemment adapter les conseils préventifs afin d'inclure des recommandations touchant ces facteurs de risque telles que la recommandation d'immunisation contre l'hépatite $B$, les risques associés à des relations sexuelles occasionnelles et comment avoir des relations sexuelles plus sûres.

Les connaissances sur les risques d'infections transmises sexuellement ne préviendront jamais toutes les infections. Des connaissances sur ce qui pousse deux personnes à avoir des relations sexuelles considérées à haut risque malgré qu'elles aient été informées seraient certainement utiles pour améliorer les activités de prévention.

Claire est une jeune médecin de famille qui vient pour un examen gynécologique au retour d'un voyage à Cuba. Elle a depuis son retour une infection vaginale qui n'a pas répondu à deux traitements antifongiques, un traitement antiparasitaire de cinq jours et une application de sept jours de crème combinée. Elle se demande si elle n'aurait pas contracté une "levure tropicale» particulière et vous demande votre avis. Elle est mariée. Elle était seule en voyage. Elle a eu une seule relation sexuelle avec un "Dieu cubain " qui devait lui donner une leçon privée de plongée. Mais l'équipement n'était pas en parfait état et il lui a plutôt fait visiter, "exclusivement » une grotte magnifique. Elle avait prévu une combinaison de latex pour la plongée, mais l'a enlevée pour la visite privée! Non, elle n'avait pas de condom. Elle n'allait pas en vacances "pour ça»! Elle est mariée et fidèle! Enfin, habituellement. Elle est vraiment très embarrassée.

Claire a contracté une gonorrhée résistante aux antibiotiques habituels. Les données sur les voyages récents ou l'origine des partenaires lors des enquêtes sur les maladies transmises sexuellement sont incomplètes ou les questions ne sont pas posées. Malgré tout, les données disponibles indiquent que, dans tous les pays industrialisés, une proportion importante des gonorrhées résistantes sont importées.

Les relations sexuelles entre partenaires consentants qui vivent en couple peuvent aussi parfois représenter une situation litigieuse en voyage. Des couples hétérosexuels de religion différente peuvent être 
harcelés dans certains pays. Ce risque est beaucoup plus important pour les couples gais. Les Affaires étrangères du Canada ont d'ailleurs émis un avis en ce sens. Même si le mariage entre personnes du même sexe est reconnu au Canada, il ne l'est pas dans plusieurs pays. Ces couples pourraient se voir refusée l'entrée dans certains pays qui n'acceptent pas l'homosexualité ou ne reconnaissent tout simplement pas ces unions. De plus, I'homosexualité est considérée illégale dans plusieurs pays du monde et une personne reconnue coupable d'activités homosexuelles illégales risque une amende, une peine d'emprisonnement, des coups de fouets, la déportation ou une sentence de mort.

Les relations sexuelles et les rencontres avec de nouveaux partenaires en voyage ont heureusement le plus souvent un dénouement positif. Les mesures de protection sont disponibles et connues. Parfois, ces rencontres se termineront même par une union et un des deux partenaires viendra habiter le pays de son conjoint.

Amalfi est mon patient de très longue date. C'est un professionnel québécois qui a beaucoup travaillé en Asie et surtout en Thaillande pour la Banque alimentaire mondiale. Il a rencontré Jade en Thaillande il y a 18 mois. Amalfi m'a appelée de Thaillande quelques jours avant son mariage, inquiet pour Jade. Son test prénuptial était positif pour le VIH. II avait utilisé un test rapide (maintenant retiré du marché au Canada) qui était négatif. II se demandait lequel des tests était erroné. II reconnaissait être incertain face à sa technique pour réaliser le test lui-même, mais n'avait pas non plus confiance au test thaillandais.

J'écoutais Amalfi et je réfléchissais. Souhaitait-il une référence pour un laboratoire fiable; des conseils sur les questions à poser à Jade pour mieux vérifier ses facteurs de risques antérieurs ; retarder son mariage; annuler son mariage ; rentrer au plus vite et oublier Jade; ou ?... Non. II savait très bien que Jade avait travaillé dans des salons de massage et des agences de call-girls. II savait qu'elle avait de fortes chances d'être infectée par le VIH. II avait même demandé avant son départ une prophylaxie post-exposition "au cas où». II voulait simplement savoir si j'acceptais de la suivre comme médecin VIH à leur arrivée au Canada. L'infection probable ne remettait rien en question pour lui. Trois ans plus tard, elle va toujours bien et ils sont les heureux parents d'une petite fille.

\section{Conclusion}

Les intervenants en médecine de voyage doivent pouvoir inclure dans leurs conseils préventifs des recommandations sur les principaux risques liés au voyage. Les risques associés aux relations sexuelles avec de nouveaux partenaires ou à l'exposition aux liquides biologiques constituent des risques suffisamment fréquents pour mériter une place dans toute consultation et, plus particulièrement, auprès des jeunes voyageurs. Le rôle de l'intervenant est d'identifier ce besoin, les risques réels qui se présenteront possiblement selon le contexte et d'offrir les conseils préventifs pertinents. Au besoin, pour des risques plus particuliers, une référence à une personne experte peu s'avérer nécessaire. Mais la majorité des situations sont simples à identifier. L'efficacité des recommandations reste à être démontrée. Aucun effet négatif n'ayant été associé à ces conseils, il est considéré pertinent d'offrir à tous les voyageurs un counselling personnalisé pour discuter des habiletés et des connaissances à appliquer pour une prévention optimale.

\section{Dominique Tessier est médecin de famille} et directrice médicale des cliniques santévoyage Médisys au Canada.

\section{Notes}

1 «La convention internationale des droits des enfants, Organisation des Nations unies, adoptée le 25 mai 2000 et entrée en vigueur le 18 janvier 2002, [www.aidh.org/DE/Conv_ Protoc_porn.htm].

2 Strategies to end the selling of innocents - A panel addressing sexual abuse, exploitation and trafficking of young girls.

Tuesday 6 June 2000, 3-5 PM Labouisse Hall, UNICEF House.

This session was chaired by UNICEF's Regional Director for Western and Central Africa, Mrs. Rima Salah. Mrs. Salah gave an introduction emphasizing the estimated number of one million children entering the commercial sex trade every year.

Ruchira Gupta, an award-winning filmmaker, showed an extract from her film, "The Selling of Innocents," which illuminated the issues very well. Afterwards she told about the Youth Career Development Programme, in Thailand, which provides vocational training at selected hotels and also gives life skill training to young girls at risk...

3 Le Monde, [http://perso.wanadoo.fr/jerome. meyrignac/tousex.html].

4 [www.unicef.ca].

\section{Bibliographie}

Correia, Jason D., Robyn T. Shafer, Vishal Patel, Kevin C. Kain, Dominique Tessier, Doug MacPherson, et Jay S. Keystone (2001), "Blood and Body Fluid Exposure as a Health Risk for International Travelers", Journal of Travel Medicine, vol. 8, n5, p. 263-266.

Elliott, L., L. Cowan, L. Gruer, et A. Hardie (1993), "Business Travel and Sexually Transmitted Diseases [letter] ", British Medical Journal, vol. 306, n6877, p. 586.

MacDougall, Laura A., Theresa W. Gyorkos, Karen Leffondré, Michal Abrahamowicz, Dominique Tessier, Brian J. Ward, et J. Dick MacLean (2001), "Increasing Referral of At-Risk Travelers to Travel Health Clinics: Evaluation of a Health Promotion Intervention Targeted to Travel Agents ", Journal of Travel Medicine, vol. 8, n 5, p. 232-242.

Mulhall, B.P., M. Hu, M. Thompson, et al., (1993), "Planned Sexual Behaviour of Young Australian Visitors to Thailand", Medical Journal of Australia, vol. 158, p. 530-535.

Poland, G.A., P.H. Axelson, et M.W. Felz (1996), "Hepatitis A and B Infections Among Expatriates in Papua New Guinea: A Missed Opportunity for Immunization", Journal of Travel Medicine, vol. 3, n 4, p. 209-213.

Samuels, M.E., C.E. Koop, et P.I. Hartstock (1991), "Single-use Syringes ", The New England Journal of Medicine, vol. 324, no 14, p. 996-997.

Steffen, R., M. Rickenbach, U. Willhelm, et al. (1987), "Health Problems After Travel to Developing Countries ", Journal of Infectious Diseases, vol. 156, p. 84-91. 